\title{
METALLICITY OF STARS AND GAS IN GALAXIES OF DIFFERENT HUBBLE TYPES
}

\author{
C. MÖLLER, U. FRITZE - V. ALVENSLEBEN AND K.J. FRICKE \\ Universitäts-Sternwarte Göttingen \\ Geismarlandstraße 11 \\ D - 37083 Göttingen, Germany \\ e-mail: cmoelle@uni-sw.gwdg.de
}

Many observations point out the great difference in metallicities determined from broad band colors of various wavelength regimes.

Our new model of chemically consistent spectrophotometric evolutionary synthesis takes into account stellar evolutionary tracks and color calibrations dependent on metallicity with $Z=10^{-4} \ldots 2 \cdot 10^{-2}$. For each of the different metallicities we include a big library of stellar spectra from $9 \cdot 10^{-3} \mu \mathrm{m}$ to $160 \mu \mathrm{m}$ computed by Kurucz (1992). Thereby we can quantitatively calculate the luminosity weighted stellar metallicities $L_{U}$ to $L_{K}$ and analyse as a function of wavelength the luminosity contributions of stellar populations with different metallicities.

Several metallicity indices are computed with the fitting functions from Gorgas et al.(1993). For various Hubble types we show the time evolution of these stellar metallicity indices $\left(\mathrm{Mg}_{2}, \mathrm{Mgb}, \mathrm{Fe} 5335, \mathrm{NaD} . ..\right)$ and compare them with the elemental ISM abundances $[\mathrm{Mg} / \mathrm{H}],[\mathrm{Fe} / \mathrm{H}]$. Theoretical calibrations for stellar indices in terms of $[\mathrm{Fe} / \mathrm{H}]$ are given and compared to previous relations.

The degree of age-metallicity-degeneracy is significantly different in various colors. Our models allow us to disentangle age and metallicity contributions at any wavelength and study their behaviour as a function of time. 